\title{
Photo-polymerisation variables influence the structure and subsequent thermal response of dental resin matrices
}

Slobodan Sirovica ${ }^{\mathrm{a}, \mathrm{b}^{*}}$, Yilan Guo ${ }^{\mathrm{c}}$, Raymond Guan ${ }^{\mathrm{c}}$, Maximilian W.A. Skoda ${ }^{\mathrm{d}}$, William M.

Palin $^{\mathrm{e}}$, Alexander P. Morrell ${ }^{\mathrm{a}, \mathrm{b}}$, Dan L. Romanyk ${ }^{\mathrm{c}, \mathrm{f}}$, Richard A. Martin ${ }^{\mathrm{a}}$ and Owen Addison ${ }^{\mathrm{b}, \mathrm{c}}$

${ }^{a}$ Aston Institute of Materials Research, School of Engineering \& Applied Science, Aston University, Birmingham, B4 7ET, UK.

${ }^{b}$ Faculty of Dental, Oral and Craniofacial Science, King's College London, Guy's Hospital, London, SE1 9RT.

${ }^{c}$ Faculty of Medicine and Dentistry, University of Alberta, Edmonton, T6G 1C9, Canada.

d ISIS Pulsed Neutron and Muon Source, Science and Technology Facilities Council, Rutherford Appleton Laboratory, Harwell Science and Innovation Campus, Didcot, OX11 OQX, UK.

${ }^{\mathrm{e}}$ College of Medical and Dental Sciences, Institute of Clinical Sciences, School of Dentistry, University of Birmingham, Mill Pool Way, Edgbaston, Birmingham, B5 7EG, UK.

${ }^{\mathrm{f}}$ Department of Mechanical Engineering, University of Alberta, Edmonton, T6G 1H9, Canada.

*Corresponding author

Slobodan Sirovica,

Faculty of Dentistry, Oral and Craniofacial Science,

King's College London,

Guy's Hospital,

London, SE1 9RT

E-Mail:

slobodan.sirovica@kcl.ac.uk 


\section{Abstract}

Objectives: The structure of the polymer phase of dental resin-based-composites is highly sensitive to photo-polymerisation variables. The objective of this study was to understand how different polymer structures, generated with different photo-polymerisation protocols, respond to thermal perturbation.

Methods: Experimental resins were prepared from a series of Bis-GMA/TEGDMA blends (40/60, 50/50 and 60/40 wt.\%), with either Camphorquinone/DMAEMA or Lucirin TPO as the photo-initiator system. Resins were photo-polymerised, in a disc geometry, at either relatively 'high' $\left(3000 \mathrm{~mW} \mathrm{~cm}{ }^{-2}\right.$ for $6 \mathrm{~s}$ ) or 'low' ( $300 \mathrm{~mW} \mathrm{~cm}{ }^{-2}$ for $60 \mathrm{~s}$ ) irradiances ensuring matched radiant exposures $\left(18 \mathrm{~J} \mathrm{~cm}^{-2}\right)$. Specimens were heated, from $20-160^{\circ} \mathrm{C}$ at a rate of $5^{\circ} \mathrm{C} \mathrm{min}^{-1}$, whilst simultaneous synchrotron X-ray scattering measurements were taken at $5^{\circ} \mathrm{C}$ increments to determine changes in polymer chain segment extension and medium-range order as a function of temperature. For each unique resin composition $(n=3)$, differential scanning calorimetry was used to measure glass transition temperatures using the same heating protocol. A paired t-test was used to determine significant differences in the glass transition temperature between irradiance protocols and photo-initiator chemistry at $a=0.05$.

Results: Resins pre-polymerised through the use of TPO and or high irradiances demonstrated a reduced rate of chain extension indicative of lower thermal expansion and a larger decrease in relative order when heated below the glass transition temperature. Above the transition temperature, differences in the rate of chain extension were negligible, but slower converted systems showed greater relative order. There was no significant difference in the glass transition temperature between different photo-initiator systems or irradiance protocols.

Significance: The evolution of chain extension and medium-range order during heating is dependent on the initial polymer structure which is influenced by photo-polymerisation variables. Less ordered systems, generated at faster rates of reactive group conversion displayed reduced chain extension below the glass transition temperature and maintained lower order throughout heating. 


\section{Introduction}

Photo-initiated dimethacrylate resin-based-composites used as dental restorative materials are subject to physical [1,2] and chemical perturbations [3,4] during their clinical service lifetime that are likely to alter the macro and micro structure of the resin phase. In vivo, a dental composite filling will experience an average intra-oral temperature of approximately $37^{\circ} \mathrm{C}$ when no other thermal stimulus is applied [5,6]. However, eating, drinking and breathing induces intra-oral thermal fluctuations which have been shown, using thermocouples, to range between $4-60^{\circ} \mathrm{C}[1,7]$ and will be partially transferred to the composite filling material. The thermal response of the resin phase will be influenced by the chemical structure of the constituent monomers [8], the degree of reactive group conversion and the polymer network structure. The formation of the polymer structure itself has been shown to be highly sensitive to photo-polymerisation variables [9-11], especially the reactive group conversion rate [12]. Previous research has investigated the dynamic response of composites to thermal perturbations by measuring dimensional changes $[13,14]$, performing mechanical tests $[15,16]$, quantifying reactive group conversion and conducting thermal analyses during heating [1622]. Assumptions have then been extrapolated about the evolving polymer structure and correlated with the initial compositional and photo-polymerisation variables. However, these approaches have failed to provide detailed insight into how the initial polymer structure changes at medium-range orders ( 4-15 $\AA$ ) during heating, which will ultimately impact on physical changes in the bulk material. The current study explores for the first time how different initial polymer structures in the most commonly encountered photo-initiated dental resin polymers, generated under different photopolymerisation protocols, are modified under thermal perturbation.

Studies have demonstrated that altering polymerisation variables, specifically by accelerating the rate of reactive group conversion using high irradiance light sources and by using more efficient photoinitiators, impacts the resultant polymer structure at a range of length scales [12]. Combined experimental and theoretical studies have suggested that during rapid polymerisation, the conversion rate substantially exceeds the relaxation time $[23,24]$ of constituent polymer structures. Consequently, 
this likely results in the jamming and clustering of growing chains, preventing efficient relaxation of the developing structure and produces a more rigid network [25] than obtained at lower polymerisation rates. Theoretical approaches [26] have demonstrated that faster polymerisation generates a polymer network with significantly greater clustering i.e. with greater spatial heterogeneity with respect to cross-link density, compared with slower rates, where reactive group conversion is more evenly distributed throughout the polymer [25]. Additionally, rapid polymerisation reduces the lifetime of propagating radical species due to bimolecular termination [27], limiting the degree of cross-linking. Recently, we have demonstrated that the conformation of polymer segments at medium-range orders, particularly the functional groups of dimethacrylate polymers, are sensitive to the conversion rate [12]. Accelerating reactive group conversion confers greater polymer segment extension in a shorter period of time. The evolving structure has insufficient time to relax and orientate to lower energy conformations [12] and consequently greater structural variability (lower order) exists in the network at medium-range length scales. Insight into the conformation of polymer segments at medium-range orders is important as these structures are constituent in cross-links and chain backbones, which play a role in determining second order phase transitions i.e. the glass transition, of the material.

Dimensional changes in the polymer phase of dental composites during heating are predominantly ascribed to structural modifications of the polymer network as the system reaches the glass transition $\left(T_{g}\right)$ region. Below $T_{g}$ the polymer network structure remains rigid, restricting polymer segmental motions [28] and demonstrates glass-like behaviour [29,30]. As the system transitions through $T_{g}$ chain molecular translations and rotations increase and the bulk material becomes rubbery [28-30]. To date, several studies have investigated the use of post-cure heating to increase the degree of reactive group conversion [16,31-37] to improve the mechanical properties of the composite material. Vibrational spectroscopy [20,31] and differential scanning calorimetry (DSC) are routinely used to measure additional cure. DSC also provides thermal information regarding phase transitions, heat and degradation and allows for differences in network architecture to be indirectly inferred based on the thermal response of the polymer as a function of the initial polymerisation variables. However, neither 
approach provides dynamic structural information of medium-range ordering, relevant to second order phase transitions, during heating. Synchrotron X-ray scattering is a powerful technique that can discern subtle structural differences within a dynamic system. Furthermore, a high flux permits time resolved measurements at time scales of the order of heating rates commonly used in conventional thermal studies of polymers [13], which would not be possible with a laboratory X-ray source.

The aim of this study was to explore how different initial polymer structures, generated by using different photo-polymerisation protocols that impact on the polymerisation reaction rate, are modified by thermal perturbation through a combination of heating, in-situ X-ray scattering and offline differential scanning calorimetry measurements. Specifically, the hypothesis tested was that, based on previous investigations, there would be a difference in polymer chain segment extension and mediumrange order as a function of temperature between systems initially polymerised at different conversion rates.

\section{Methods}

\subsection{Preparation of photo-polymerisable dimethacrylate resin blends}

Two dimethacrylate monomers, bisphenol-A-glycidyl-methacrylate (Bis-GMA) and triethyleneglycoldimethacrylate (TEGDMA) (Sigma-Aldrich, Dorset, UK) were proportioned in 60/40, 50/50 and 40/60 (Bis-GMA/TEGDMA) weight percentage (wt. \%) ratios and combined to produce mixes. The viscosity of the blend increases with the proportion of Bis-GMA [38] (see supplementary material,

Figure S1). The monomer blends were combined with a photo-initiator as either 0.2 wt.\% Camphorquinone (CQ) with 0.8 wt.\% of its tertiary amine N,N-dimethylaminoethyl-methacrylate (DMAEMA) or 1 wt.\% of the Type 1 photo-initiator, Lucirin-TPO (TPO) (Sigma-Aldrich, Dorset, UK), which does not require a co-initiator. The proportioned monomers and photo-initiators were homogenised in a glass beaker using a magnetic stirrer at $50 \pm 1{ }^{\circ} \mathrm{C}$ for 30 mins in dark conditions. 
The resultant resin blends were stored in dark conditions in sealed containers at $4 \pm 1^{\circ} \mathrm{C}$ prior to further use. Resin disc-shaped specimens were fabricated by filling a polyvinylsiloxane mould (11 $\mathrm{mm}$ diameter and $1 \mathrm{~mm}$ in depth) covered with a $1 \mathrm{~mm}$ thickness glass slide to minimise the formation of an oxygen inhibition layer. Specimens were photo-polymerised using an EMS Swiss master light curing unit (EMS OPTIDENT, electro medical systems, Nyon Switzerland) placed normal to and in contact with the glass slide. The curing unit has a spectral output range of $390-550$ $\mathrm{nm}$ to provide significant overlap with the absorption peaks of the Camphorquinone $(470 \mathrm{~nm})$ and Lucirin TPO $(381 \mathrm{~nm})$ photo-initiators. For each unique composition, the resin was either photopolymerised at relatively 'high' $\left(3000 \mathrm{~mW} \mathrm{~cm} \mathrm{c}^{-2}\right.$ for $\left.6 \mathrm{~s}\right)$ or 'low' $\left(300 \mathrm{~mW} \mathrm{~cm}^{-2}\right.$ for $\left.60 \mathrm{~s}\right)$ irradiances to introduce a range of reactive group conversion rates whilst ensuring matched radiant exposures (18 $\mathrm{J} \mathrm{cm}^{-2}$ ). The resultant specimens were fabricated two weeks prior to any measurements and were stored in lightproof conditions in sealed containers at $4 \pm 1{ }^{\circ} \mathrm{C}$ to remove post-cure variability attributed to further light activation $[39,40]$.

\subsection{FT-NIR spectroscopy measurements}

Fourier Transform Near Infra-red (FT-NIR) spectroscopy was used to quantify the rate of reactive group conversion between relatively high $\left(3000 \mathrm{~mW} \mathrm{~cm}^{-2}\right)$ and low $\left(300 \mathrm{~mW} \mathrm{~cm} \mathrm{c}^{-2}\right)$ irradiances to confirm differences in rate as a function of irradiance protocol, for selected monomer blends, according to a previously reported method [12]. Briefly, optical (emitting and receiving) fibers (0.6 mm core diameter) (Hellma Analytics, Essex, UK) were placed either side of the liquid resin sample, which was held within a polyvinylsiloxane annulus (11 $\mathrm{mm}$ diameter and $1 \mathrm{~mm}$ in depth), at a $45^{\circ}$ incline relative to the sample face and connected to a Nicolet 6700 spectrometer (Thermo Scientific, Warrington, UK). During photo-polymerisation, NIR spectra were collected in transmission mode using a white light source and an InGaAs detector. Real-time NIR spectra (4000-10000 $\left.\mathrm{cm}^{-1}\right)$ were collected $10 \mathrm{~s}$ prior to irradiance and thereafter during polymerisation in transmission mode $\left(4 \mathrm{~cm}^{-1}\right.$ spectral resolution) with an integration time of $0.3 \mathrm{~s}$, whilst photo-polymerising the resin sample according to protocol described in section 2.1. Three measurements were conducted for each unique 
blend for both irradiance protocols. Data were baseline corrected using Omnic software (Omnic Specta software, version 8.0, Thermo Fisher Scientific, Oxford, UK). Reactive group conversion was determined from the percentage decrease in the aliphatic $\mathrm{C}=\mathrm{CH}_{2} \mathrm{IR}$ absorption band $\left(6164 \mathrm{~cm}^{-1}\right)$ i.e. the conversion of $\mathrm{C}=\mathrm{C}$ to $\mathrm{C}-\mathrm{C}$ bonds, located within the functional end groups of the Bis-GMA and TEGDMA monomers. The rate of conversion was calculated as the first derivative of conversion with respect to time.

\subsection{Synchrotron $X$-ray scattering measurements}

Synchrotron X-ray scattering experiments were performed on the I16 beamline [41] at the Diamond Light Source (Oxford, UK). An incident X-ray energy of $12 \mathrm{keV}$ was used corresponding to a wavelength $(\lambda)$ of $1.033 \AA$, with a beam size of $30 \mu \mathrm{m}$ (vertical) $\times 200 \mu \mathrm{m}$ (horizontal) defined by vacuum tube slits. Measurements were carried out in air with light excluded.

A bespoke heating module was used to increase the temperature of pre-polymerised BisGMA/TEGDMA resin disc specimens produced according to the previously described methods, whilst undertaking simultaneous X-ray scattering measurements (Figure 1). The heating module consisted of two aluminium plates $(25 \times 25 \mathrm{~mm}$ area) with a circular aperture ( $3 \mathrm{~mm}$ diameter), to allow for the transmission of X-rays, fixed in contact with and perpendicular to a kinematic heating platform. The temperature of the heating platform was remotely controlled via a cryogenic temperature modulator (Model 3.8, Lake Shore Cryotonics, Elliot Scientific Ltd, Hertfordshire, UK). Polymer disc specimens were held in tight contact between two aluminium plates. The temperature of the test specimen was increased through heat transfer from the kinematic stage to the aluminium plates and finally to the resin disc, at a rate of $5^{\circ} \mathrm{C} \mathrm{min}^{-1}$. Three thermocouples were placed in contact with the kinematic stage, aluminium plates and the resin disc respectively, to measure the temperature of each component and ensure a constant heating rate. The heating module and housed sample were orientated with the annulus of the aluminium plate normal to the path of the impinging X-rays.

$\mathrm{X}$-ray scattering measurements were taken at $5^{\circ} \mathrm{C}$ increments, from 20 to $160^{\circ} \mathrm{C}$, to characterise structural changes as a function of temperature. X-ray scattering data were collected using a 2D area 
detector (Pilatus 100K) with a $487 \times 195$ pixel format $\left(\right.$ pixel size $=172 \times 172 \mu \mathrm{m}^{2}$ ) fixed to a two theta detector arm. The detector was placed $470 \mathrm{~mm}$ behind the sample and collected scattering data at angles of $2 \theta=7,13$ and 20 to cover a $q$ range of 0.2 to $1.8 \AA^{-1}$ where $q=4 \pi \sin \theta / \lambda$. Scattering data were collected with a $1 \mathrm{~s}$ count time and a $2.7 \mathrm{~ms}$ readout time. The transmitted beam was also recorded at each angle and temperature increment using a photodiode to correct for changes to the sample density and thickness. Due to the high user demand of synchrotron facilities which limits the duration of experiments, measurements for each unique sample composition were not repeated.

The contribution of the X-ray beam to the measurements i.e. beam damage was assessed by conducting observations for over $30 \mathrm{~min}$ for the pre-polymerised samples at ambient temperature. To aid data analysis, measurements were taken for direct beam, empty sample containers and a silver behenate calibration standard. Scattering patterns were background corrected and normalised to the incident monitor intensity. Data were integrated over $360^{\circ}$ to produce a $1 \mathrm{D}$ output and were subsequently fitted with a pseudo-Voigt model to determine peak positions and changes in the peak full width at half maximum (FWHM) as a function of temperature using the Dawn software package (version 2.6.0) $[42,43]$. The peak position(s) correspond to the measured average correlation length(s) whilst the relative structural order representing the distribution of correlation lengths was calculated from changes in the FWHM of the scattering peak(s).

\subsection{Thermal analysis}

To correlate changes in X-ray scattering during heating with structural transitions, the glass transition temperature $\left(T_{g}\right)$ and post-cure exotherm for polymerised resin specimens were measured using a differential scanning calorimeter (DSC Q1000, TA Instruments, Ontario, Canada). Samples (n=3, per composition) of approximately $5 \mathrm{mg}$ were heated, within hermetically sealed aluminium pans (DSC Consumables Incorporated, Minnesota, USA), through a scanning range of $20-160^{\circ} \mathrm{C} \pm 0.05^{\circ} \mathrm{C}$ at a rate of $5^{\circ} \mathrm{C} \min ^{-1}$ under a nitrogen atmosphere. The $T_{g}$ values were obtained from the inflection point in the thermogram (Universal Analysis 2000, TA Instruments, Ontario, Canada), as measured from 
the extensions of the pre- and post-transition baselines. Reaction exotherm was obtained from the integrated area of exothermic peaks in the thermogram (Universal Analysis 2000, TA Instruments, Ontario, Canada).

\subsection{Statistical analysis}

For each resin monomer composition, a one tailed t-test was used to determine differences in the rate of reactive group conversion between identical blends polymerised at identical irradiances, for samples using either CQ or TPO as photo-initiator. A one-way ANOVA and post-hoc Tukey tests $(\alpha=0.05)$ were also used to identify differences in $T_{g}$ and exotherm values between samples photopolymerised at different conversion rates. Descriptive analysis was conducted on X-ray scattering data and qualitatively correlated with DSC data to aid interpretation. All statistical analysis was performed in the R programming language (version 3.1.3, 2015, R Foundation). 


\section{Results}

\subsection{De-convolving the scattering signal}

Figure 2(a) shows representative 1-D X-ray scattering spectra for the same sample (40/60 wt.\%, BisGMA/TEGDMA, TPO photo-initiator, irradiance $=3000 \mathrm{mWcm}^{-2}$ ) at $20^{\circ} \mathrm{C}$ and $160^{\circ} \mathrm{C}$. A broad scattering feature is located at $2 \theta \sim 10^{\circ}$ and shifts to lower scattering angles with increasing temperature. Deconvolution of the X-ray scattering spectrum (Figure 2(b)) reveals a high-agreement fit for two scattering peaks located at $2 \theta \approx 10.3^{\circ} \pm 0.0045^{\circ}$ and $17.4^{\circ} \pm 0.036^{\circ}$, corresponding to correlation lengths in real space of approximately $5.55 \AA$ and $3.43 \AA$ respectively. When the resin is incrementally heated, the principle scattering peak located at $10.3^{\circ}$ shifts to lower values of $2 \theta$, indicative of an increase in the correlation length. Additionally, the width of the peak fluctuates as a function of temperature indicating temperature dependent changes in medium-range order. The scattering feature at $\sim 17.4^{\circ}$ displays a small shift to wider scattering angles upon heating, corresponding to a decrease in this correlation length, whilst the FWHM value increases linearly (see supplementary material, Figure S2).

\subsection{The effect of temperature on correlation length}

Figure 3 illustrates the relative change in the principle correlation length for all resin samples as a function of temperature, where the correlation length corresponds to the peak position of the scattering feature at $2 \theta \sim 10.3^{\circ}$ in Figure 2 at each temperature increment. It can be seen that all samples demonstrate a subtle two stage increase in the relative change in the correlation length, with an inflection point located at $\sim 45-55^{\circ} \mathrm{C}$, as the temperature is increased. Prior to the inflection point, the relative change in the correlation length is approximately linear with respect to temperature and the rate of increase is $0.0110 \pm 0.0048$ and $0.0076 \pm 0.0011 \%{ }^{\circ} \mathrm{C}^{-1}$ for the CQ and TPO based systems, respectively. After the inflection point, the rate of relative change in the correlation length increases for all samples and remains linear with values of $\sim 0.025 \%{ }^{\circ} \mathrm{C}^{-1}(\mathrm{CQ}=0.0248 \pm 0.0038 \%$ ${ }^{\circ} \mathrm{C}^{-1}$, TPO $\left.=0.0250 \pm 0.0018 \%{ }^{\circ} \mathrm{C}^{-1}\right)$, regardless of differences in the co-monomer ratio, photo- 
initiator chemistry or the irradiance protocol used. The total relative increase in the correlation length i.e. between the highest and lowest temperature values is $\sim 3 \%$ for all samples except for ' $40 / 60 \mathrm{wt}$. $\% 300 \mathrm{~mW} \mathrm{~cm}^{-2}(\mathrm{CQ})$, which shows larger initial and final gains in the correlation length during heating, however the steady state percentage increase in correlation length is similar to other resins. Differences between CQ and TPO initiated systems for the rate and total relative change in the correlation length are negligible after the inflection point.

\subsection{The effect of temperature on medium-range order}

All resins show undulations in the FWHM value of the principal scattering peak shown in Figure 2 as a function of temperature (see supplementary material, Figure S3). A narrowing of the scattering peak represents an increase in relative order, whilst a broadening of this peak relates to reduced order. The CQ photo-initiated resins display smaller values of FWHM compared with the TPO based systems for all compositions and equivalent irradiance protocols at all temperatures i.e. CQ photoinitiated resins demonstrate greater medium-range order. Resins initiated with TPO display offsets in the FWHM which are maintained throughout heating and can be ranked by the relative viscosities of monomer blends (ratio of Bis-GMA/TEGDMA) (see supplementary material, Figures S1,S3). Differences in FWHM as a function of irradiance protocol and monomer ratios are negligible in the CQ initiated systems.

Relative changes in order as a function of temperature are shown in Figure 4, where values of order correspond to the change in the FWHM of the scattering peak shown in Figure 2, relative to the initial FWHM value for each temperature increment. Local minima and maxima in relative order are observed between $45-65^{\circ} \mathrm{C}$ and $80-110^{\circ} \mathrm{C}$ respectively. The minimum displays a decrease in order of $\sim 2.5 \%$ for TPO initiated and $\sim 1 \%$ for the CQ initiated systems. For TPO initiated systems, the changes in relative order tend to zero between $80-120^{\circ} \mathrm{C}$, whilst CQ systems display a gain of $\sim 1 \%$. Raising the temperature above $120^{\circ} \mathrm{C}$ conferred a decrease in order for TPO based resins whilst CQ systems displayed minor or negligible changes. 


\subsection{Thermal analysis}

Figure 5 shows a representative DSC thermogram for a 40/60 wt. \% (Bis-GMA/TEGDMA) TPO initiated resin heated through a temperature range of $20-160^{\circ} \mathrm{C}$ at a rate of $5^{\circ} \mathrm{C} \mathrm{min}^{-1}$. Two prominent thermal features are observed; a glass transition located at approximately $50^{\circ} \mathrm{C}$ and a broad exothermic peak which starts and ends at $\sim 60$ and $130^{\circ} \mathrm{C}$ respectively. The average $T_{g}$ values and standard deviations for all resin compositions and irradiances are summarised in Table 1. There is generally no significant difference between $T_{g}$ values for CQ and TPO initiated resins for either identical blend composition or irradiance protocol $(\mathrm{p}<0.05)$. Additionally, resins initiated with CQ typically displayed greater exotherm post $T_{g}$ (Table 2) compared with TPO based systems, however differences between irradiances for a given photo-initiator system were not significant $(\mathrm{p}<0.05)$.

\subsection{Polymerisation kinetics}

Figure 6 shows the rate of reactive group conversion for 60/40, 50/50 and 40/60 wt.\% (BisGMA/TEGDMA) resins polymerised at either $300 \mathrm{mWcm}^{-2}(60 \mathrm{~s})$ or $3000 \mathrm{mWcm}^{-2}(6 \mathrm{~s})$ for CQ and TPO initiated systems. It can be seen that the rate of conversion is significantly greater for TPO based systems for a given irradiance and blend compared with CQ initiated resins $(\mathrm{p}<0.05)$. Additionally, TPO resins irradiated at $300 \mathrm{mWcm}^{-2}$ display similar or greater conversion rates than CQ systems polymerised at $3000 \mathrm{mWcm}^{-2}$. Greater conversion rates are also observed in blends with a larger content of TEGDMA.

\section{Discussion}

\subsection{Origin and mechanism of temperature dependent structural changes}

Heating induced an increase in the correlation length of the X-ray scattering feature observed at $2 \theta \sim$ $10.3^{\circ}(\sim 5.55 \AA)$ in Bis-GMA/TEGDMA resins (Figure 2(a)). It has previously been demonstrated that this scattering peak corresponds to a correlation length originating primarily from the methacrylic functional end groups of the constituent monomers and the ether backbone of TEGDMA [12], 
constituent in cross-linking structures. It must also be acknowledged that a portion of this correlation length will originate from the polymer chain backbone. Increases in the correlation length are predominantly ascribed to thermally driven polymer segmental motions, i.e. heat generates a more energetic system, conferring chain extension and modifying structural order.

The scattering peak located at $2 \theta \sim 17.3^{\circ}$ is believed to originate from the Van der Waals separation distance of neighbouring unreacted monomers and trapped radical species. This is based on the magnitude of the correlation length closely matching previously reported values for the Van der Waals separation distance for these systems $[44,45]$ as well as the insensitivity of the correlation length and order to second order phase transitions (see supplementary material, Figure S2). Correlation lengths from either neighbouring cross-links or nearest neighbour covalent bonds are ruled out as these correlation lengths would be expected to increase upon thermal perturbation. Decreases in the Van der Waals separation distance as a function of temperature are attributed to the expansion of the polymer network during heating, increasing the effective volume of the polymer and raising the mobility of the radical species, allowing them to come closer together. Variations to the Van der Waals separation distance of unreacted monomers and radicals are not the focus of this study as these components do not contribute to second order phase transitions.

\subsection{The effect of temperature on polymer segment chain extension and order}

The relative change in the correlation length and medium-range order demonstrate an inflection point and minima respectively at $\sim 45-55^{\circ} \mathrm{C}$ (Figures 3 and 4), as a function of temperature. The inflection and minima are attributed to the glass transition region for these dimethacrylate systems [46] and is consistent with DSC measurements presented in this study (Figure 5 and Table 1) and with previous investigations for Bis-GMA/TEGDMA resin formulations $[13,22]$. Similar behaviour with regards to the minima in medium-range order as a function of temperature for other polymer systems have also been reported [46]. As the system tends towards $T_{g}$ only localised vibrational and rotational motions of bonds and monomer segments are permitted [47], generating a wider range of structural 
conformations associated with increased thermal motions, producing the observed increase in the correlation length (chain extension) and decrease in order.

Post- $T_{g}$, the rate of relative increase in the correlation length is greater than before $T_{g}$ and order increases between $60-120^{\circ} \mathrm{C}$ (Figure 5). The increase in the rate of chain extension is ascribed to larger scale molecular motions within polymer segments, cross-links and chain backbones which are permitted above $T_{g}$. Subsequent increases in order post- $T_{g}$ are likely due to additional structural reorientation and reactive group conversion, implied by the exothermic feature in the DSC thermograms between $60-120^{\circ} \mathrm{C}$ (Figure 5 and Table 2). This provides further support to the hypothesis that the minima in order at $45-55^{\circ} \mathrm{C}$ corresponds to $T_{g}$ as further cure may only occur once $T_{g}$ has been reached [22] to permit sufficient mobility for formerly trapped radical species $[16,22]$. Additional polymerisation will also confer an increase in the observed correlation length. This is not necessarily attributed to true chain extension but will represent a gradual increase in the contribution of longer correlation lengths (arising from increasing $\mathrm{C}=\mathrm{C}$ to $\mathrm{C}-\mathrm{C}$ conversion) to the average scattering signal. Additional reactive group conversion, indicated by the exotherm in DSC thermograms (Table 2), is likely small as the rate of chain extension post- $T_{g}$ is linear (linear fits $=\mathrm{R}^{2}$ $>0.95$ ). Bond degradation is not believed to play a role in any non-linear behaviour of the polymer as there is no indication of degradation in the DSC data and onset temperatures of degradation have been reported to be much greater than those used in this study $[20,48]$.

Below the glass transition region, slower converted systems (typically CQ and relatively low irradiance, Figure 6) show a greater rate of relative change in the correlation length and a smaller decrease in relative order when heated. As the system transitions through $T_{g}$, differences in the rate and total relative change in the principal correlation length with respect to temperature are negligible between systems generated at higher and lower rates of reactive group conversion. Conversely, relative order remains greater and becomes positive in more slowly converted systems. This behaviour is attributed to differences in the initial medium-range structure of the polymer network, prior to heating, as a function of the initial reactive group conversion rate. Accelerating reactive group conversion using high irradiance light sources and efficient photo-initiator chemistry (Figure 6), has 
been reported to generate lower structural order at medium-range length scales and an extended chain conformation, which is frozen into the network [12].

Therefore below $T_{\mathrm{g}}$, systems converted relatively fast (TPO and higher irradiances, Figure 6) possess a less uniform structure $[12,25,27,49]$ with an extended chain segment conformation, which is more easily disturbed and less able to extend and reorientate under thermal perturbation to relieve thermal stresses. Above the glass transition, comparable rates and total magnitudes of polymer chain segment extension for systems polymerised at different rates of conversion are ascribed to similar rotational and translational freedom at these relatively short length scales for the constituent chemical bonds within the methacrylic end groups, ether backbone of TEGDMA and chain backbones. The gain in order observed in relatively slowly converted systems post- $\mathrm{T}_{g}\left(80-110^{\circ} \mathrm{C}\right)$, is likely due to greater thermally driven post-cure. DSC traces show a greater exotherm feature after the glass transition point for CQ initiated resins indicative of greater reactive group conversion $[16,18,19,50]$ (Figure 5 and Table 2). Further conversion will constrain polymer segment motions and increase structural order [51]. However, the contribution of thermal cure is likely to be small as chain extension remains linear within this temperature range (Figure 3). However, post-cure may not entirely explain the greater gain in order for more slowly converted systems as the total gain in order is positive. This may therefore suggest minor structural re-orientation to a more ordered state permitted by a more uniform initial structure.

It must be acknowledged that a deficiency of this study is that changes in the polymer structure have only been observed as the temperature increases. In vivo thermal variations are typically experienced in cycles and it is unknown how the polymer structure is modified at medium-range orders as the polymer cools. Repeated heating and cooling may conceivably minimise or even remove structural differences at medium-range orders between polymers generated at different conversion rates in a process akin to annealing. Further work is therefore required to elucidate the relationship between initial polymerisation variables, polymer microstructure and the thermal response of the system. 


\section{Conclusions}

In this study in-situ X-ray scattering and DSC measurements have been used to understand how different polymer structures of dimethacrylate resins, generated through different rates of reactive group conversion, are modified by thermal perturbations. Heating confers an increase in polymer chain segment extension and modifies medium-range order, both of which are sensitive to second order phase transitions and the initial polymer structure, which in turn varies as a function of the conversion rate. Below the glass transition temperature, chain extension is greater in more slowly converted systems whilst above the transition point differences are negligible. Less ordered systems, generated at faster conversion rates maintain lower order throughout heating, compared with more slowly converted systems; this is ascribed to a lower ordered initial conformation frozen into the polymer network which inhibits structural modification. This study demonstrates how photopolymerisation variables, specifically conversion rate, and the initial polymer conformation impact on the thermal response of the polymer structure.

\section{Author contributions}

S.S., O.A., R.A.M., and M.W.A.S. conceived the experiment. S.S, M.W.A.S and A.P.M performed Xray scattering measurements. Y.G., R.G. and D.L.R. conducted DSC analysis. S.S., O.A. and W.M.P. performed FT-NIR measurements. S.S., O.A., R.A.M., and M.W.A.S. interpreted the data and drafted the manuscript. All authors revised the manuscript prior to submission.

\section{Funding}

The following funding is acknowledged: Science and Technology Facilities Council (award no. ST/L502510/1) and the Royal College of Surgeons of England Small Grants Scheme (Faculty of Dentistry). 


\section{Notes}

The authors declare no competing financial interests.

\section{Acknowledgements}

The authors would like to thank Diamond Light Source for beam time (proposal MT15319-1) and the staff of the I16 beam line for their assistance with data collection. 


\section{References}

[1] Drummond JL. Degradation, fatigue, and failure of resin dental composite materials. J Dent Res 2008;87:710-9. doi:10.1177/154405910808700802.

[2] Lutz F, Krejci I, Barbakow F. Chewing Pressure us. Wear of Composites and Opposing Enamel Cusps. J Dent Res 1992;71:1525-9. doi:10.1177/00220345920710081201.

[3] Bettencourt AF, Neves CB, de Almeida MS, Pinheiro LM, Oliveira SA e, Lopes LP, et al. Biodegradation of acrylic based resins: A review. Dent Mater 2010;26:e171-80. doi:https://doi.org/10.1016/j.dental.2010.01.006.

[4] Ferracane JL. Hygroscopic and hydrolytic effects in dental polymer networks. Dent Mater 2006;22:211-22. doi:https://doi.org/10.1016/j.dental.2005.05.005.

[5] Gale MS, Darvell BW. Thermal cycling procedures for laboratory testing of dental restorations. J Dent 1999;27:89-99. doi:https://doi.org/10.1016/S0300-5712(98)00037-2.

[6] Brown AC, Goldberg MP. Surface temperature and temperature gradients of human teeth in sith Archives of Oral. vol. 11. 1966. doi:10.1016/0003-9969(66)90199-3.

[7] Minami H, Hori S, Kurashige H, Murahara S, Muraguchi K, Minesaki Y, et al. Effects of Thermal Cycling on Surface Texture of Restorative Composite Materials. Dent Mater J 2007;26:316-22. doi:10.4012/dmj.26.316.

[8] Sideridou I, Tserki V, Papanastasiou G. Effect of chemical structure on degree of conversion in light-cured dimethacrylate-based dental resins. Biomaterials 2002;23:1819-29. doi:10.1016/S0142-9612(01)00308-8.

[9] Calheiros FC, Kawano Y, Stansbury JW, Braga RR. Influence of radiant exposure on contraction stress, degree of conversion and mechanical properties of resin composites. Dent Mater 2006;22:799-803. doi:https://doi.org/10.1016/j.dental.2005.11.008. 
[10] Stansbury JW. Dimethacrylate network formation and polymer property evolution as determined by the selection of monomers and curing conditions. Dent Mater 2012;28:13-22. doi:https://doi.org/10.1016/j.dental.2011.09.005.

[11] Ogunyinka A, Palin WM, Shortall AC, Marquis PM. Photoinitiation chemistry affects light transmission and degree of conversion of curing experimental dental resin composites. Dent Mater 2007;23:807-13. doi:https://doi.org/10.1016/j.dental.2006.06.016.

[12] Sirovica S, W. A. Skoda M, Podgorski M, B. J. Thompson P, Palin W, Guo Y, et al. Structural Evidence That the Polymerization Rate Dictates Order and Intrinsic Strain Generation in Photocured Methacrylate Biomedical Polymers. Macromolecules 2019;52:5377-88. doi:10.1021/acs.macromol.9b00133.

[13] Sideridou I, Achilias DS, Kyrikou E. Thermal expansion characteristics of light-cured dental resins and resin composites. Biomaterials 2004;25:3087-97. doi:https://doi.org/10.1016/j.biomaterials.2003.09.078.

[14] Versluis A, Douglas WH, Sakaguchi RL. Thermal expansion coefficient of dental composites measured with strain gauges. Dent Mater 1996;12:290-4. doi:https://doi.org/10.1016/S01095641(96)80036-4.

[15] Lovell LG, Lu H, Elliott JE, Stansbury JW, Bowman CN. The effect of cure rate on the mechanical properties of dental resins. Dent Mater 2001;17:504-11. doi:10.1016/S01095641(01)00010-0.

[16] Vaidyanathan J, Vaidyanathan TK, Wang Y, Viswanadhan T. Thermoanalytical characterization of visible light cure dental composites. J Oral Rehabil 1992;19:49-64. doi:10.1111/j.1365-2842.1992.tb01590.x.

[17] Miyazaki CL, Medeiros IS, Matos J do R, Rodrigues Filho LE. Thermal characterization of dental composites by TG/DTG and DSC. J Therm Anal Calorim 2010;102:361-7. doi:10.1007/s10973-010-0739-3. 
[18] Almeida CC, Mothé CG. Characterization of dental composites by thermal analysis, infrared spectroscopy and scanning electron microscopy. J Therm Anal Calorim 2009;97:585. doi:10.1007/s10973-009-0359-y.

[19] Sampath S, R G. Thermal studies on different classes of clinical dental composites. vol. 111. 2013. doi:10.1007/s10973-012-2243-4.

[20] Bernardi MIB, Rojas SS, Andreeta MRB, de Rastelli ANS, Hernandes AC, Bagnato VS. Thermal analysis and structural investigation of different dental composite resins. $\mathrm{J}$ Therm Anal Calorim 2008;94:791. doi:10.1007/s10973-008-8820-x.

[21] Dionysopoulos P, Watts DC. Dynamic mechanical properties of an inlay composite. J Dent 1989;17:140-4. doi:https://doi.org/10.1016/0300-5712(89)90110-3.

[22] Leprince JG, Leveque P, Nysten B, Gallez B, Devaux J, Leloup G. New insight into the "depth of cure" of dimethacrylate-based dental composites. Dent Mater 2012;28:512-20. doi:https://doi.org/10.1016/j.dental.2011.12.004.

[23] Anseth KS, Kline LM, Walker TA, Anderson KJ, Bowman CN. Reaction Kinetics and Volume Relaxation during Polymerizations of Multiethylene Glycol Dimethacrylates. Macromolecules 1995;28:2491-9. doi:10.1021/ma00111a050.

[24] Cook WD. Photopolymerization kinetics of oligo(ethylene oxide) and oligo(methylene) oxide dimethacrylates. J Polym Sci Part A Polym Chem 1993;31:1053-67. doi:10.1002/pola.1993.080310428.

[25] Sarkar S, Baker PJ, Chan EP, Lin-Gibson S, Chiang MYM. Quantifying the sensitivity of the network structure and properties from simultaneous measurements during photopolymerization. Soft Matter 2017;13:3975-83. doi:10.1039/C7SM00419B.

[26] Camboni F, Koher A, Sokolov IM. Diffusion of small particles in a solid polymeric medium. Phys Rev E 2013;88:22120. doi:10.1103/PhysRevE.88.022120. 
[27] Feng L, Carvalho R, Suh BI. Insufficient cure under the condition of high irradiance and short irradiation time. Dent Mater 2009;25:283-9. doi:https://doi.org/10.1016/j.dental.2008.07.007.

[28] Bower DI, editor. Morphology and motion. An Introd. to Polym. Phys., Cambridge: Cambridge University Press; 2002, p. 117-61. doi:10.1017/CBO9780511801280.006.

[29] Bower DI, editor. Mechanical properties I - time-independent elasticity. An Introd. to Polym. Phys., Cambridge: Cambridge University Press; 2002, p. 162-86. doi:10.1017/CBO9780511801280.007.

[30] Bower DI, editor. Mechanical properties II - linear viscoelasticity. An Introd. to Polym. Phys., Cambridge: Cambridge University Press; 2002, p. 187-219. doi:10.1017/CBO9780511801280.008.

[31] Trujillo M, Newman SM, Stansbury JW. Use of near-IR to monitor the influence of external heating on dental composite photopolymerization. Dent Mater 2004;20:766-77. doi:https://doi.org/10.1016/j.dental.2004.02.003.

[32] Lecamp L, Youssef B, Bunel C, Lebaudy P. Photoinitiated polymerization of a dimethacrylate oligomer: Part 3. Postpolymerization study. Polymer (Guildf) 1999;40:6313-20. doi:https://doi.org/10.1016/S0032-3861(99)00042-7.

[33] Jancar J, Wang W, Dibenedetto AT. On the heterogeneous structure of thermally cured bisGMA/TEGDMA resins. J Mater Sci Mater Med 2000;11:675-82. doi:10.1023/A:1008999023271.

[34] Tanoue N, Matsumura H, Atsuta M. Comparative evaluation of secondary heat treatment and a high intensity light source for the improvement of properties of prosthetic composites. J Oral Rehabil 2000;27:288-93. doi:10.1046/j.1365-2842.2000.00507.x.

[35] Wendt SL, Leinfelder KF. The Clinical Evaluation of Heat-Treated Composite Resin Inlays. J Am Dent Assoc 1990;120:177-81. doi:https://doi.org/10.14219/jada.archive.1990.0038. 
[36] Loza-Herrero MA, Rueggeberg EA, Caughman WE, Schuster GS, Lefebvre CA, Gardner EM. Effect of Heating Delay on Conversion and Strength of a Post-cured Resin Composite. J Dent Res 1998;77:426-31. doi:10.1177/00220345980770021201.

[37] Peutzfeldt A, Asmussen E. The effect of postcuring on quantity of remaining double bonds, mechanical properties, and in vitro wear of two resin composites. J Dent 2000;28:447-52. doi:https://doi.org/10.1016/S0300-5712(00)00021-X.

[38] Ellakwa A, Cho N, Lee IB. The effect of resin matrix composition on the polymerization shrinkage and rheological properties of experimental dental composites. Dent Mater 2007;23:1229-35. doi:https://doi.org/10.1016/j.dental.2006.11.004.

[39] Leung RL, Fan PL, Johnston WM. Post-irradiation Polymerization of Visible Light-activated Composite Resin. J Dent Res 1983;62:363-5. doi:10.1177/00220345830620031201.

[40] Tarumi H, Imazato S, Ehara A, Kato S, Ebi N, Ebisu S. Post-irradiation polymerization of composites containing bis-GMA and TEGDMA. Dent Mater 1999;15:238-42. doi:https://doi.org/10.1016/S0109-5641(99)00040-8.

[41] Collins SP, Bombardi A, Marshall AR, Williams JH, Barlow G, Day AG, et al. Diamond Beamline I16 (Materials \& Magnetism). AIP Conf Proc 2010;1234:303-6. doi:10.1063/1.3463196.

[42] Basham M, Filik J, Wharmby MT, Chang PCY, El Kassaby B, Gerring M, et al. Data Analysis WorkbeNch (DAWN). J Synchrotron Radiat 2015;22:853-8. doi:10.1107/S1600577515002283.

[43] Filik J, Ashton A, C Y Chang P, A Chater P, J Day S, Drakopoulos M, et al. Processing twodimensional X-ray diffraction and small-angle scattering data in DAWN 2. vol. 50. 2017. doi:10.1107/S1600576717004708.

[44] Peutzfeldt A. Resin composites in dentistry: the monomer systems. Eur J Oral Sci 
1997;105:97-116. doi:10.1111/j.1600-0722.1997.tb00188.x.

[45] Rueggeberg FA. From vulcanite to vinyl, a history of resins in restorative dentistry. J Prosthet Dent 2002;87:364-79. doi:10.1067/mpr.2002.123400.

[46] Frick B, Richter D, Ritter C. Structural Changes near the Glass Transition-Neutron Diffraction on a Simple Polymer. Europhys Lett 1989;9:557-62. doi:10.1209/0295-5075/9/6/011.

[47] Hoffman JD. Anelastic and dielectric effects in polymeric solids, N. G. McCrum, B. E. Read, and G. Williams, Wiley, New York, 1967. pp. 617. J Appl Polym Sci 1969;13:397. doi:10.1002/app.1969.070130214.

[48] Achilias DS, Karabela MM, Sideridou ID. Thermal degradation of light-cured dimethacrylate resins: Part I. Isoconversional kinetic analysis. Thermochim Acta 2008;472:74-83. doi:https://doi.org/10.1016/j.tca.2008.02.004.

[49] Elliott JE, Lovell LG, Bowman CN. Primary cyclization in the polymerization of bis-GMA and TEGDMA: a modeling approach to understanding the cure of dental resins. Dent Mater 2001;17:221-9. doi:https://doi.org/10.1016/S0109-5641(00)00075-0.

[50] Dewaele M, Asmussen E, Peutzfeldt A, Munksgaard EC, Benetti AR, Finné G, et al. Influence of curing protocol on selected properties of light-curing polymers: Degree of conversion, volume contraction, elastic modulus, and glass transition temperature. Dent Mater 2009;25:1576-84. doi:https://doi.org/10.1016/j.dental.2009.08.001.

[51] Darvell BW. Chapter 5 - Acrylic. In: Darvell BW, editor. Materials Science for Dentistry. ninth ed. Woodhead Publishing; 2009, p. 108-27. doi:https://doi.org/10.1533/9781845696672.108. 


\section{Figures}

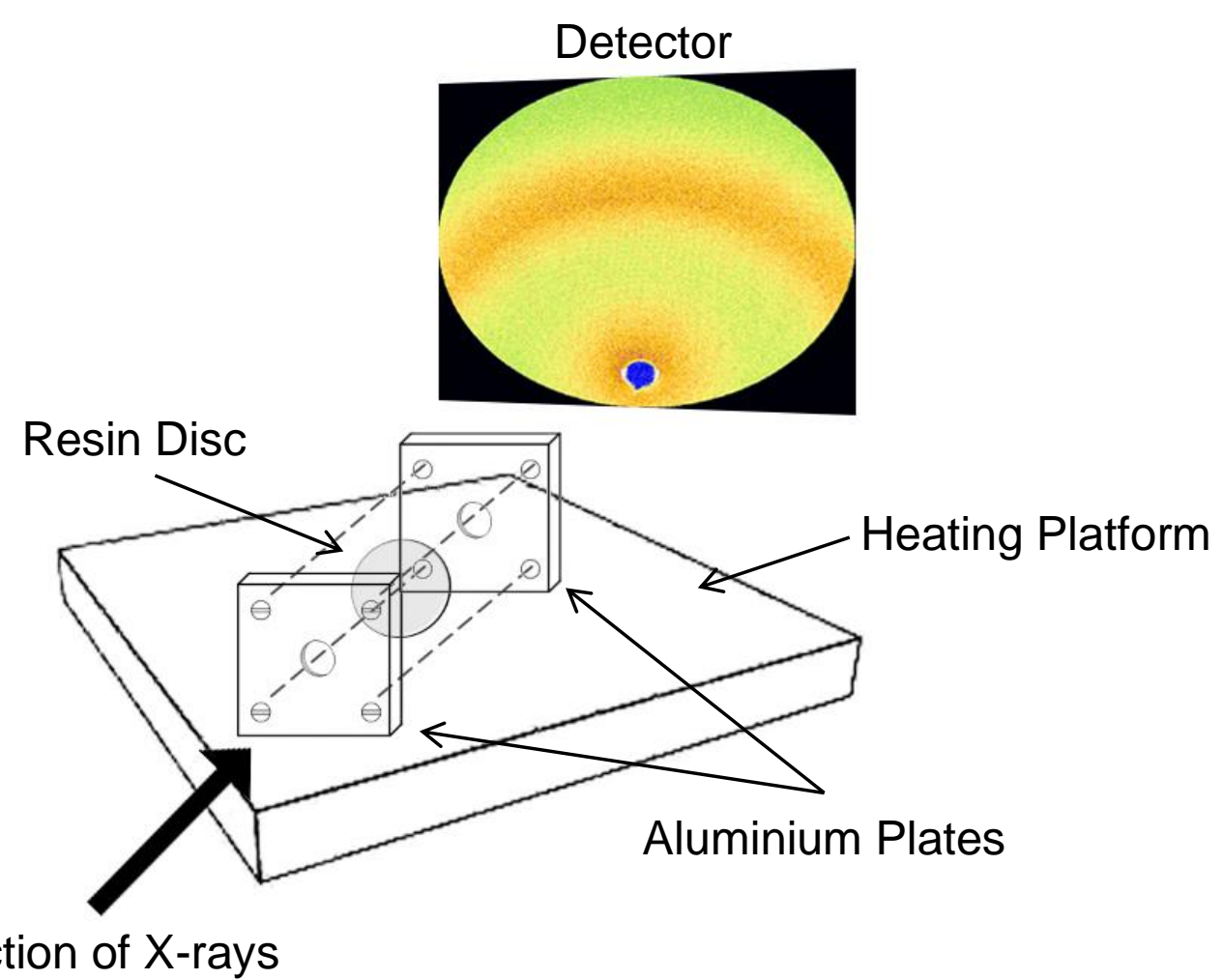

Figure 1. Simplified experimental setup at the I16 beamline (Diamond Light Source) with an example 2D diffraction image (image for illustrative purposes, collected at BM28, ESRF). A pre-polymerised resin disc specimen is stored in good contact between two aluminium plates normal to impinging X-rays. The aluminium housing is mounted in contact and perpendicular to a kinematic heating platform, used to increase the temperature of the resin specimen through contact with the aluminium plates. Thermocouples (not shown) are attached to the heating platform, aluminium housing and the resin specimen to monitor the temperature and heating rate. X-ray scattering measurements are taken at $5^{\circ} \mathrm{C}$ intervals between $20-160^{\circ} \mathrm{C}$. 

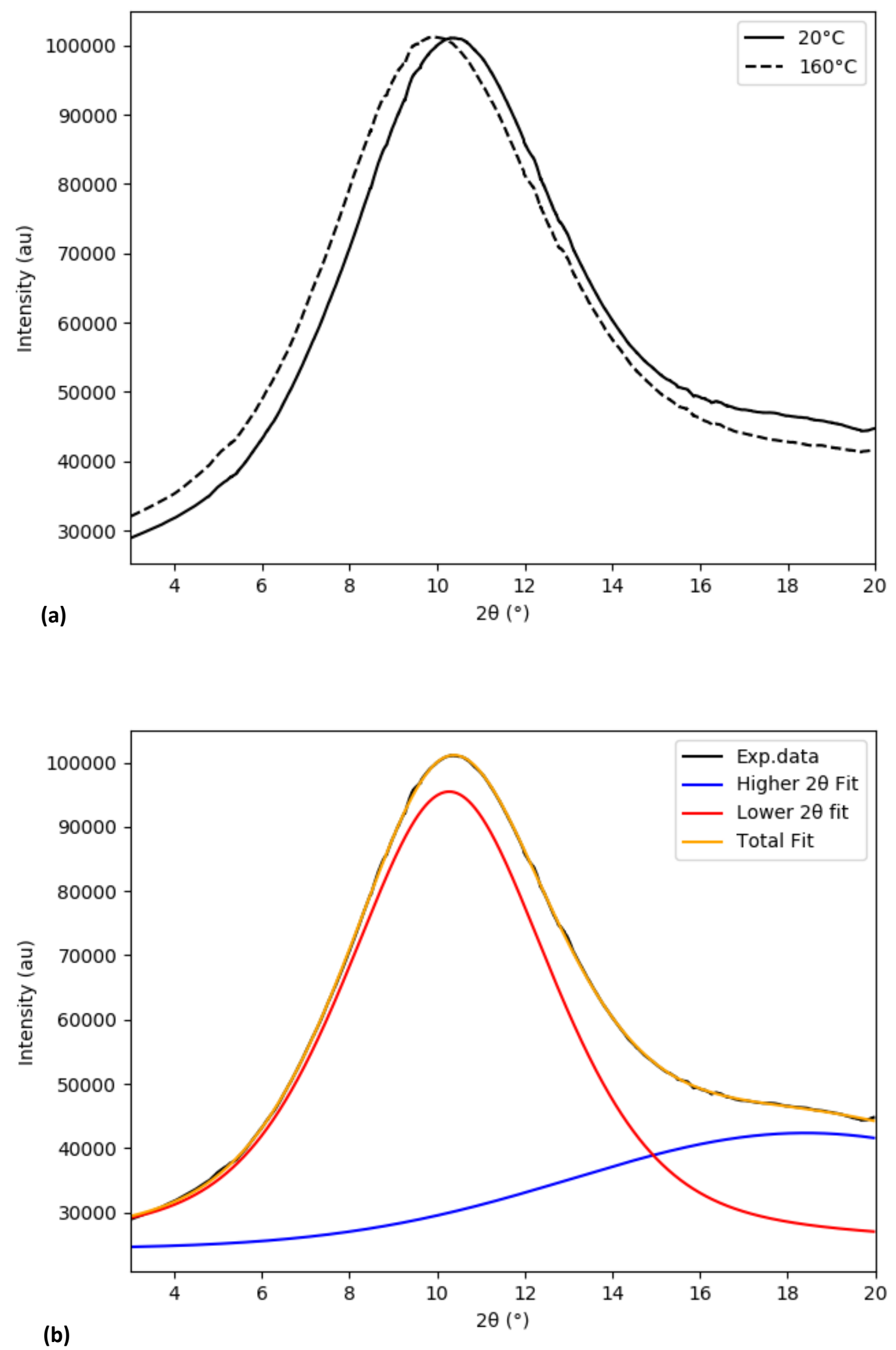

Figure 2. (a) X-ray scattering spectrum of a 40/60 wt.\%, Bis-GMA/TEGDMA, TPO photo-initiator (irradiance = $3000 \mathrm{mWcm}^{-2}$ ) at $20^{\circ} \mathrm{C}$ and $160^{\circ} \mathrm{C}$. The principal scattering feature is initially located at $\sim 10.3^{\circ}$ at a temperature of $20{ }^{\circ} \mathrm{C}$ (solid black line) and shifts to lower values of $2 \theta$ when heated up to $160{ }^{\circ} \mathrm{C}$ (broken black line). Fluctuations in the peak width are also observed as a function of temperature. (b) Deconvolution of the scattering feature reveals two underlying broad peaks located at approximately 10.3 and $17.3^{\circ}$. 

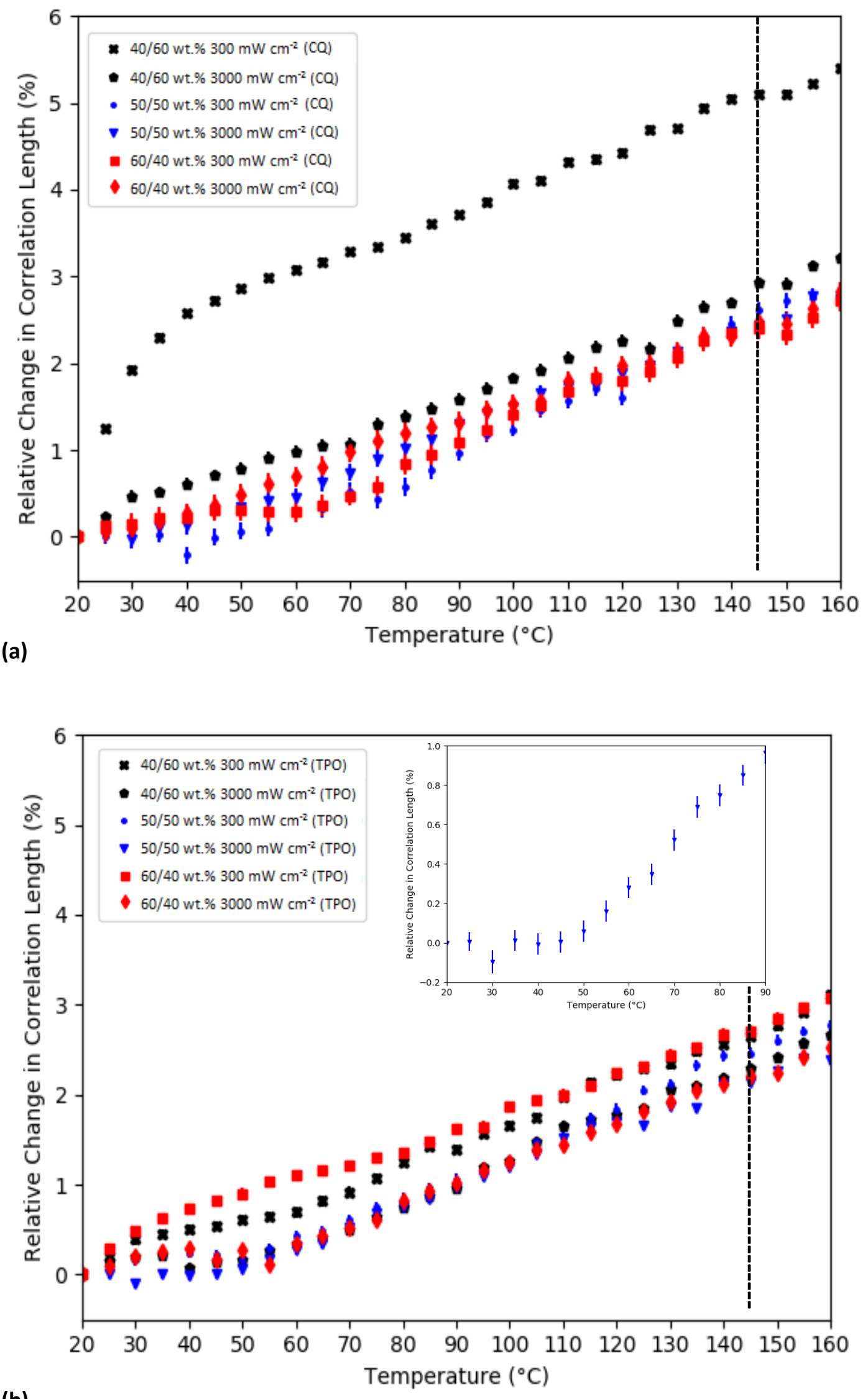

(b)

Figure 3. Relative percentage change in the principal correlation length as a function of temperature for (a) CQ and (b) TPO initiated resins. Here, error bars are shown but are typically smaller than the plotting symbol size. Error values were obtained from the propagation of errors of fitting uncertainties. (inset) Inflection point in the relative change in the correlation length with associated error bars. Data to the right of the broken vertical line represent points where the heating platform could not maintain the heating rate due to thermal losses, but where data was still collected at the respective temperatures. 


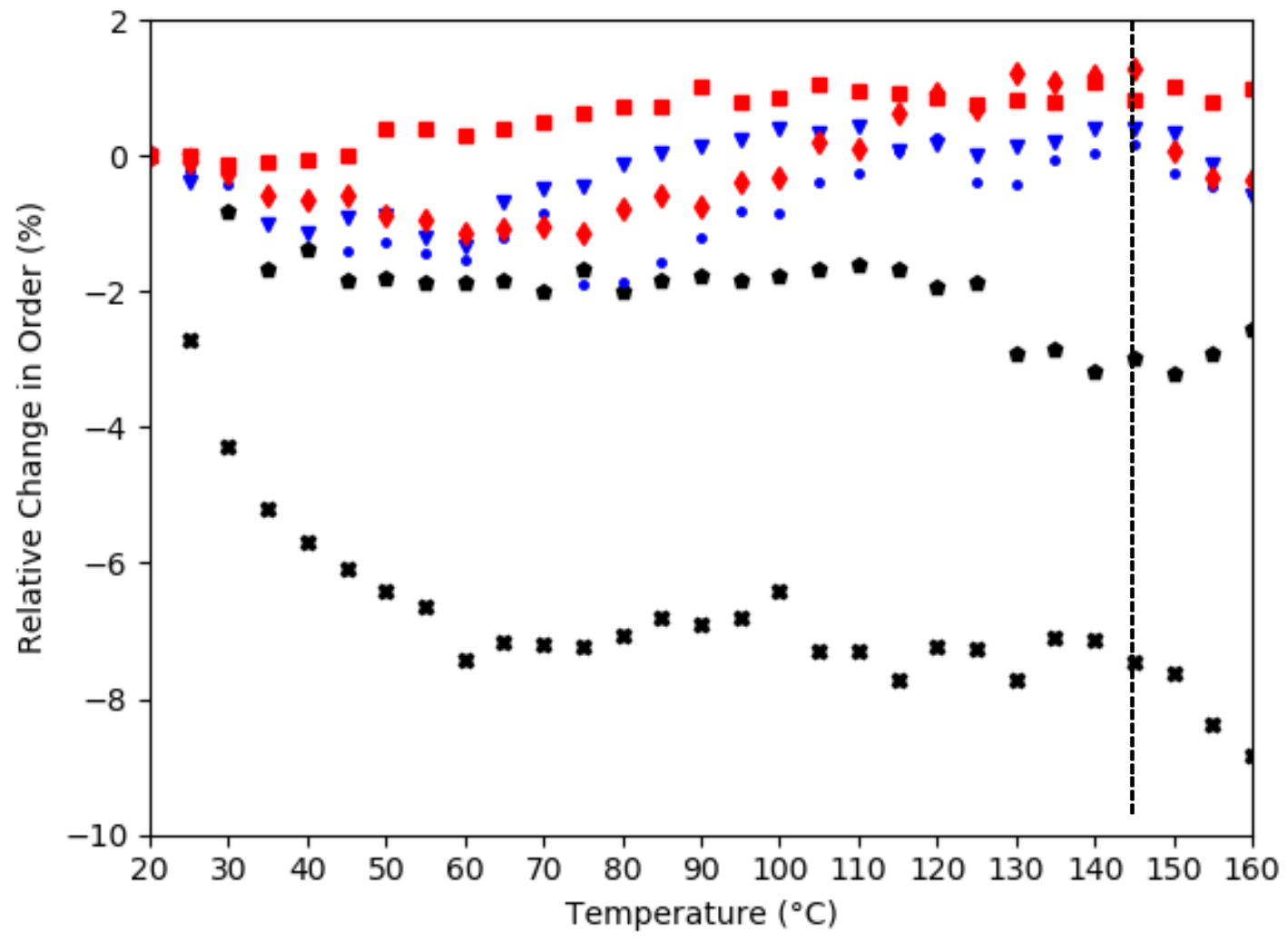

(a)

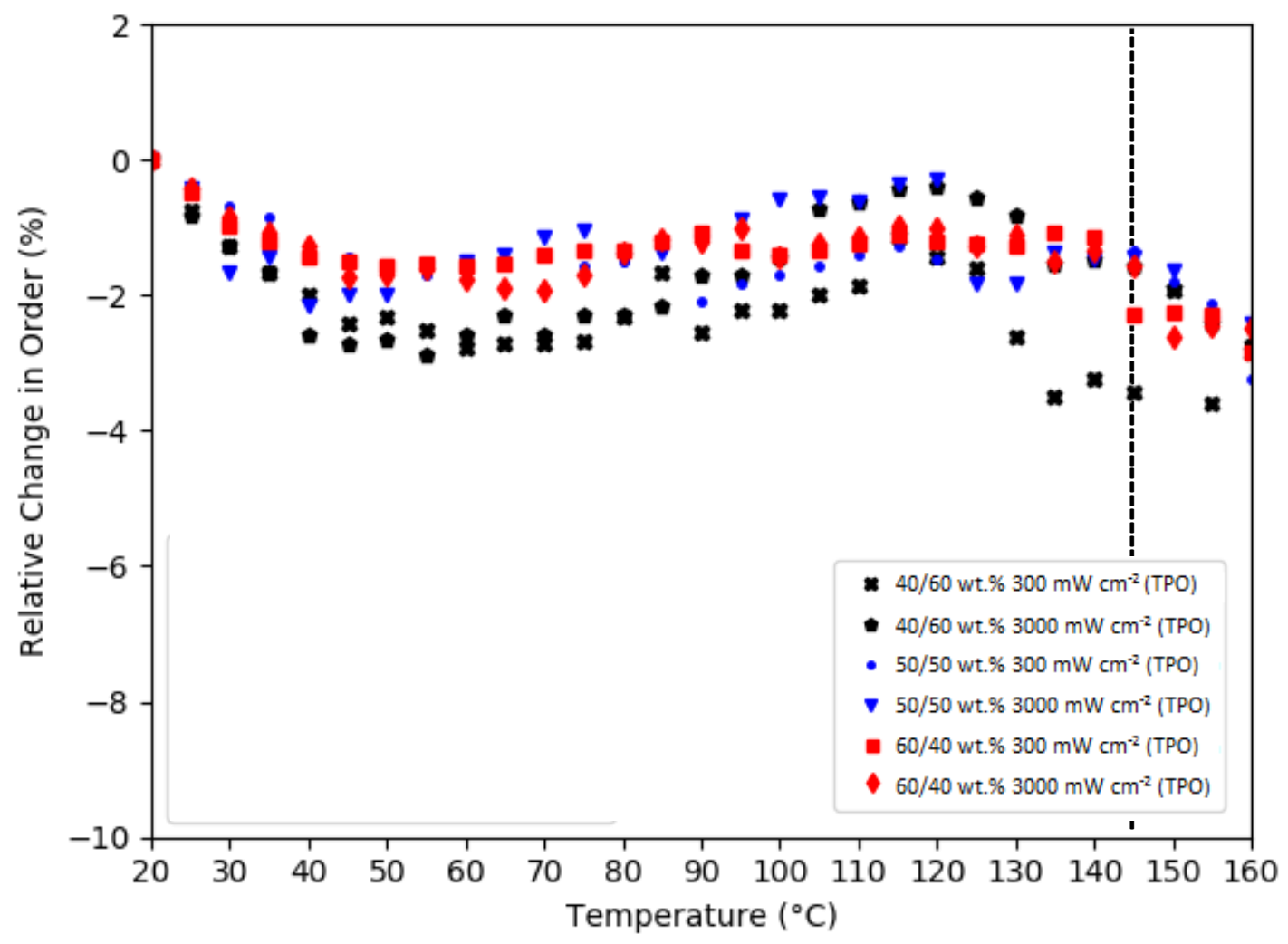

(b)

Figure 4. Relative change in order as a function of temperature for (a) CQ and (b) TPO initiated resins. Data to the right of the broken vertical line represent points where the heating platform could not maintain the heating rate due to thermal losses, but where data was still collected at the respective temperatures. 


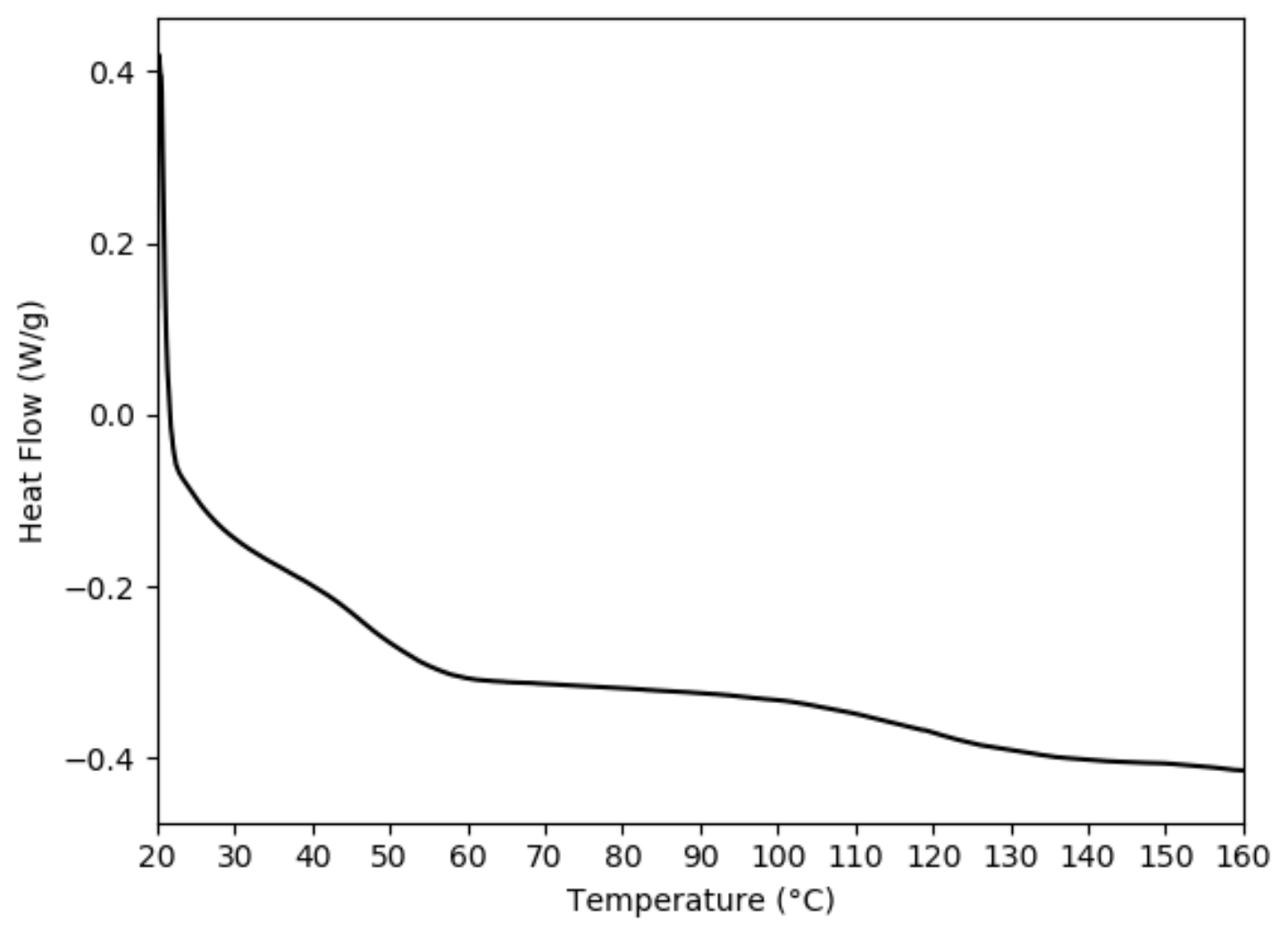

Figure 5. A representative DSC thermogram for a 40/60 wt.\% (Bis-GMA/TEGDMA) resin initiated with TPO. A glass transition region is observed at $\sim 46^{\circ} \mathrm{C}$ whilst an exotherm is seen between $60-130{ }^{\circ} \mathrm{C}$.

Table 1 - Mean glass transition temperatures $\left(T_{g}\right)$ for each unique resin composition with associated standard deviations. Common superscripts denote non-significant differences within columns and rows, within the same Bis-GMA/TEGDMA blend $(p<0.05)$.

\begin{tabular}{|c|c|c|}
\hline \multirow{2}{*}{$\begin{array}{c}\text { Resin } \\
\text { composition }\end{array}$} & $300 \mathrm{~mW} \mathrm{~cm}^{-2}$ & $3000 \mathrm{~mW} \mathrm{~cm}^{-2}$ \\
\hline & $T_{g}\left({ }^{\circ} \mathrm{C}\right)$ & $T_{g}\left({ }^{\circ} \mathrm{C}\right)$ \\
\hline $40 / 60 \mathrm{CQ}$ & $53.01 \pm 6.28^{\mathrm{a}}$ & $45.91 \pm 2.22^{\mathrm{a}}$ \\
\hline 40/60 TPO & $48.12 \pm 1.27^{a}$ & $46.05 \pm 1.18^{a}$ \\
\hline $50 / 50 \mathrm{CQ}$ & $46.97 \pm 1.58^{*}$ & $45.14 \pm 2.72^{*}$ \\
\hline 50/50 TPO & $46.88 \pm 4.92^{*}$ & $50.35 \pm 1.75^{\dagger}$ \\
\hline $60 / 40 \mathrm{CQ}$ & $49.62 \pm 3.65^{\#}$ & $46.46 \pm 1.83^{\#}$ \\
\hline 60/40 TPO & $50.54 \pm 4.62^{\#}$ & $49.73 \pm 1.14^{\#}$ \\
\hline
\end{tabular}


Table 2 - Mean exotherm, post $T_{g}$, for each unique resin composition with associated standard deviations.

Common superscripts denote non-significant differences within columns and rows, within the same Bis-GMA/TEGDMA blend ( $p<0.05$ ).

\begin{tabular}{|c|c|c|}
\hline $\begin{array}{c}\text { Resin } \\
\text { composition }\end{array}$ & $\frac{300 \mathrm{~mW} \mathrm{~cm}^{-2}}{(\mathrm{~J} / \mathrm{g})}$ & $\frac{3000 \mathrm{~mW} \mathrm{~cm}^{-2}}{(\mathrm{~J} / \mathrm{g})}$ \\
\hline $40 / 60 \mathrm{CQ}$ & $5.46 \pm 2.66^{a}$ & $5.21 \pm 1.36^{a}$ \\
\hline 40/60 TPO & $3.86 \pm 1.70^{\mathrm{a}}$ & $4.12 \pm 0.43^{a}$ \\
\hline $50 / 50 \mathrm{CQ}$ & $5.32 \pm 1.46^{*}$ & $3.88 \pm 1.39^{*}$ \\
\hline 50/50 TPO & $3.98 \pm 1.78^{*}$ & $1.63 \pm 0.96^{*}$ \\
\hline $60 / 40 \mathrm{CQ}$ & $4.08 \pm 2.54^{\#}$ & $4.81 \pm 2.70^{\#}$ \\
\hline 60/40 TPO & $1.90 \pm 0.91^{\#}$ & $6.20 \pm 0.85^{\#}$ \\
\hline
\end{tabular}




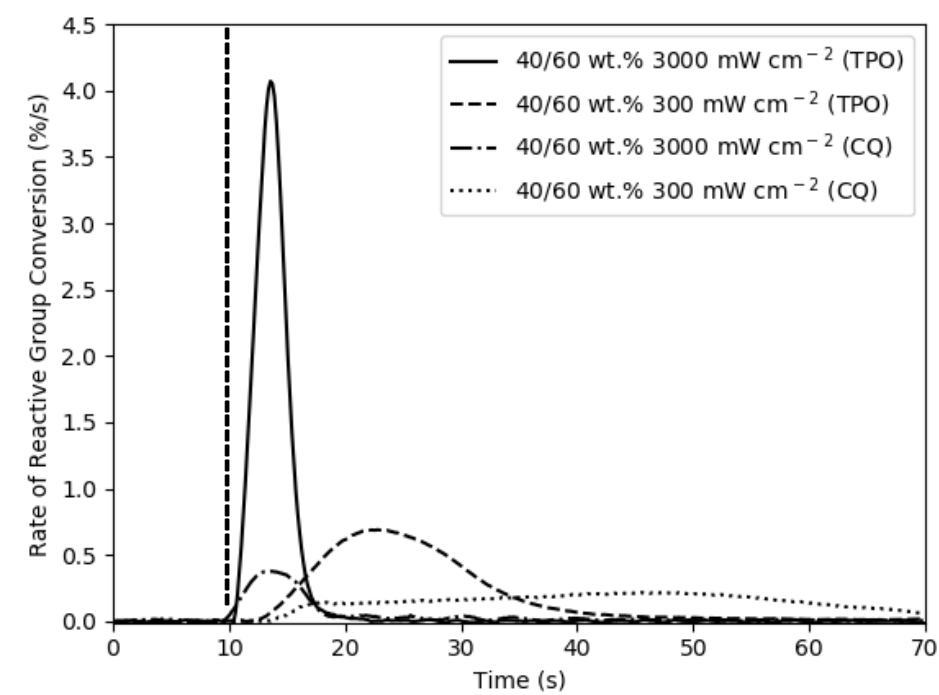

(a)

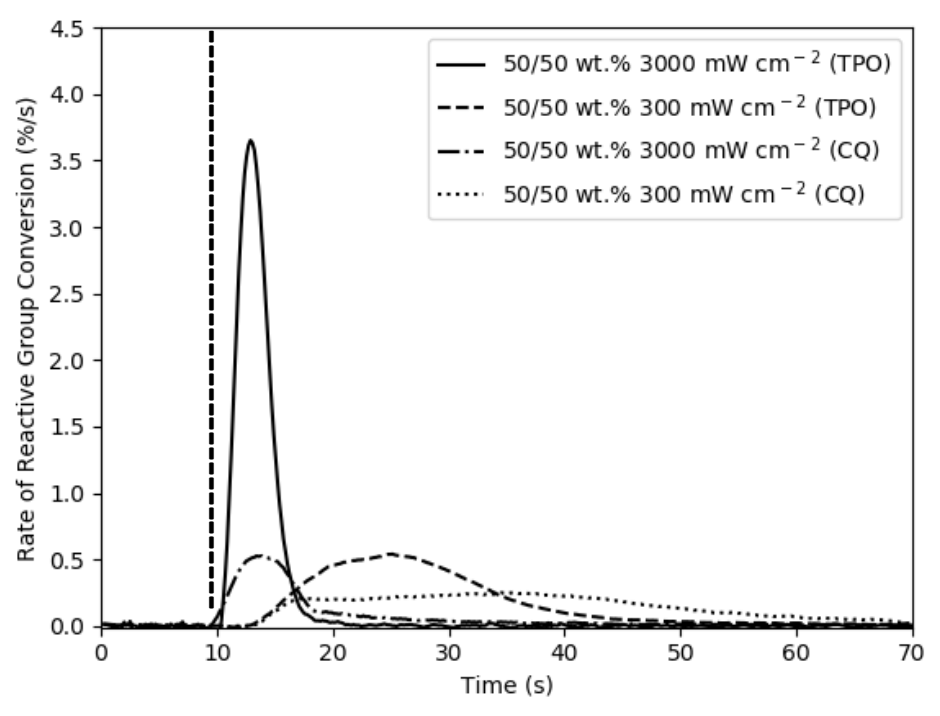

(b)

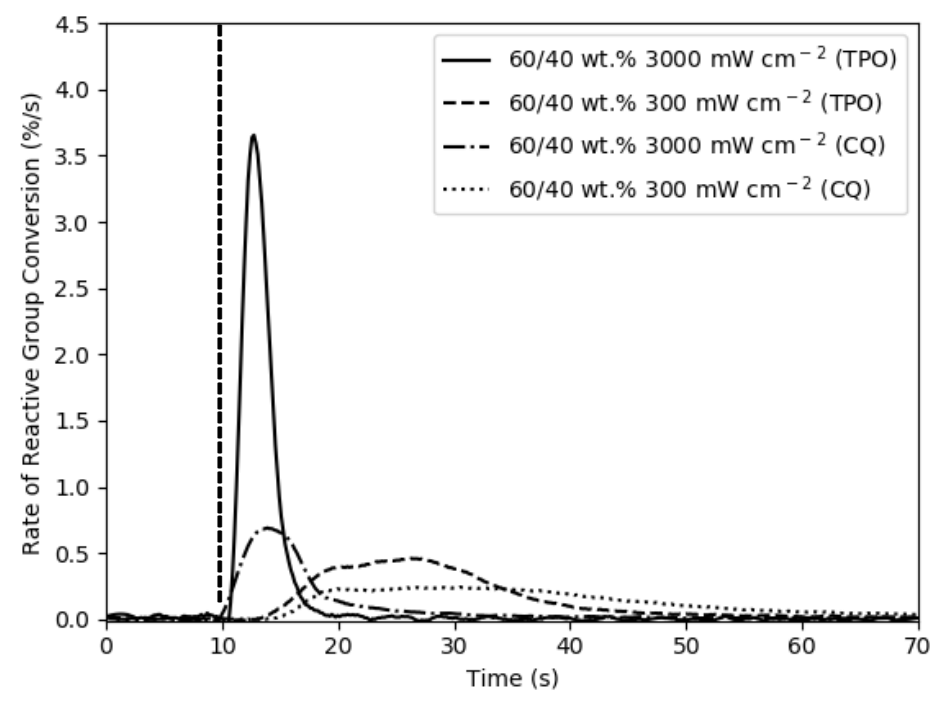

(c)

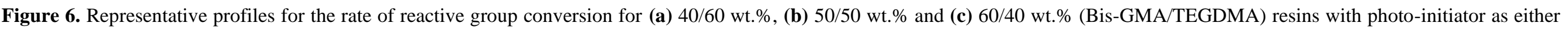
CQ or TPO and polymerised at irradiances of $300 \mathrm{mWcm}^{-2}$ or $3000 \mathrm{mWcm}^{-2}$. The broken vertical line denotes the onset of irradiance. 


\section{Supplementary material}

\section{Supplementary methods}

\section{Viscosity analysis}

The viscosities of Bis-GMA/TEGDMA wt. \% monomer blends, as a function of Bis-GMA content (20,30,40,50,60,70 and 100 wt.\%), were measured using a rheometer (AR 2000, TA Instruments, Ontario, Canada). Measurements for each unique monomer blend composition $(n=20)$ were performed at $25^{\circ} \mathrm{C} \pm 0.1^{\circ} \mathrm{C}$ using a Peltier controlled temperature plate at a shear rate of $51.6 \mathrm{~s}^{-1}$. 


\section{Supplementary results}

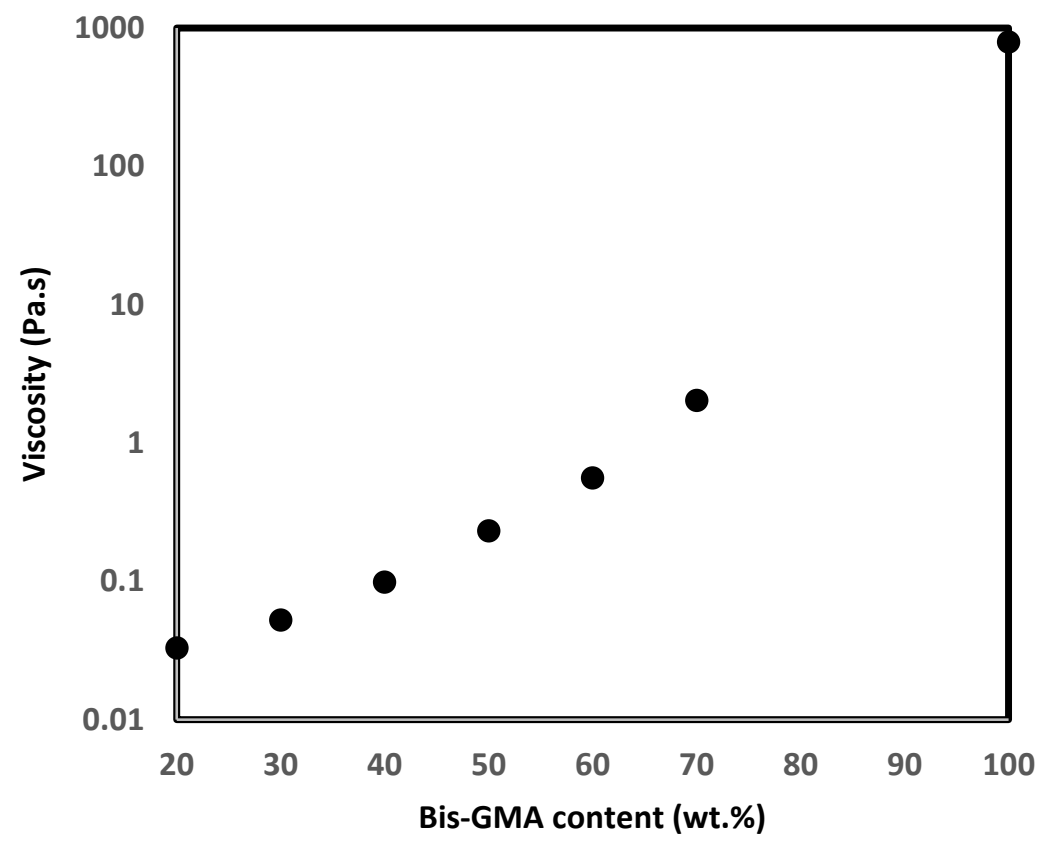

Figure S1. Viscosity of Bis-GMA/TEGDMA wt.\% monomer blends as a function of the Bis-GMA content (wt.\%). Error bars are shown but are smaller than the size of the plotting symbols. 


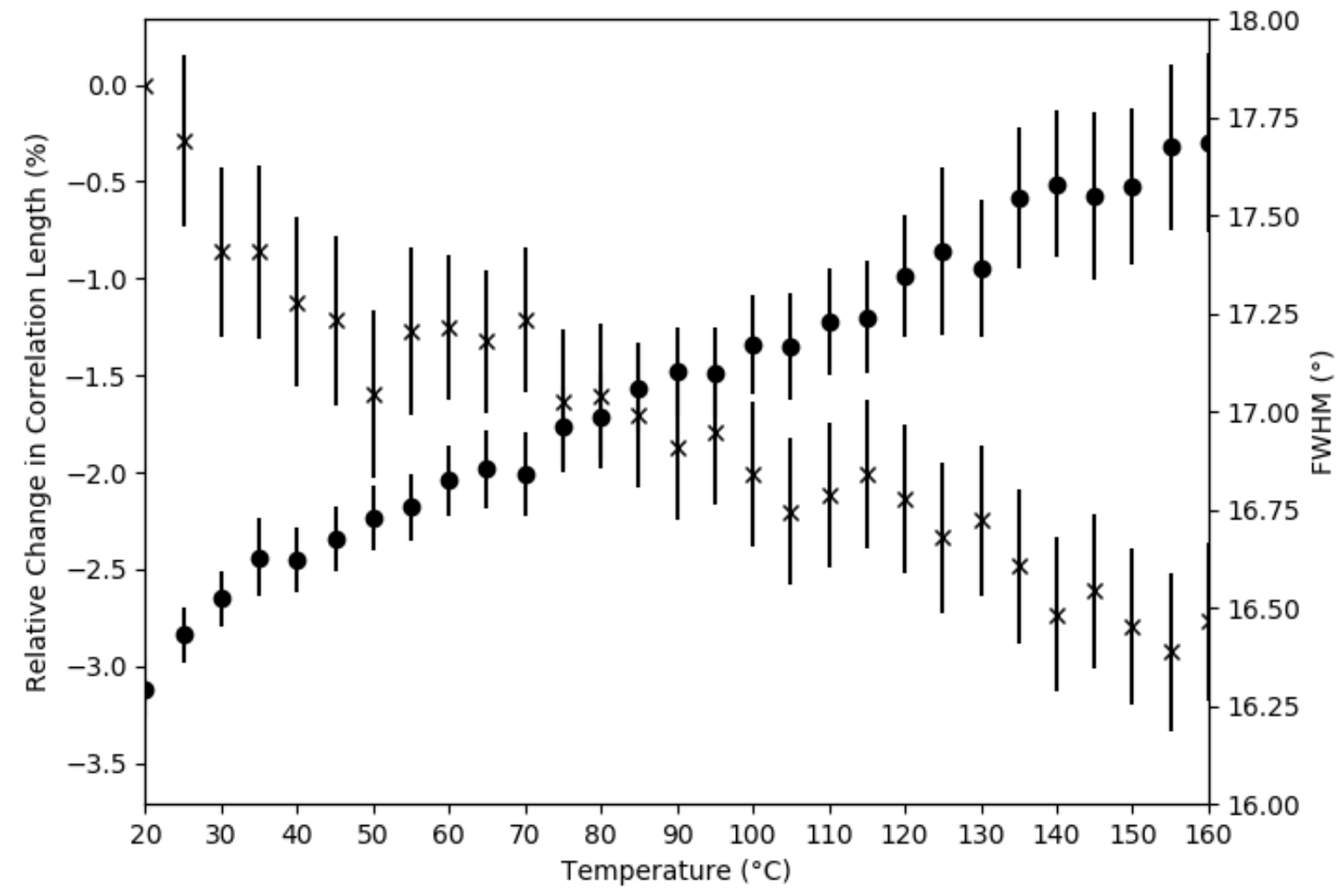

Figure S2. A representative plot of the relative change in the correlation length $(x)$ and FWHM $(\bullet)$ of the broad scattering peak located at $\sim 17.4^{\circ}$, as a function of temperature, for a 40/60 wt.\% (Bis-GMA/TEGDMA) $\mathrm{CQ}$ resin. Error bars were obtained via propagation of errors from peak fitting uncertainties. 


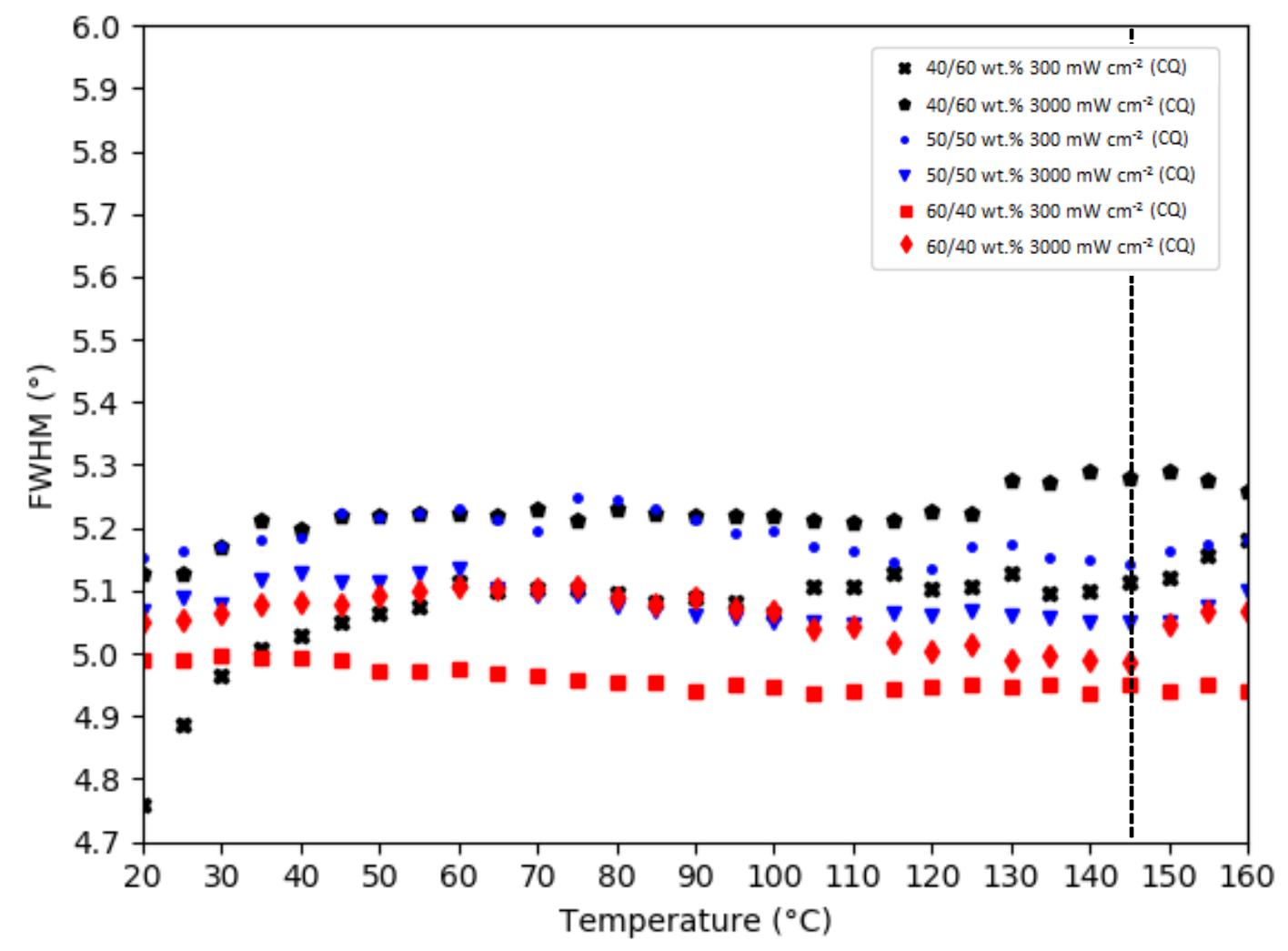

(a)

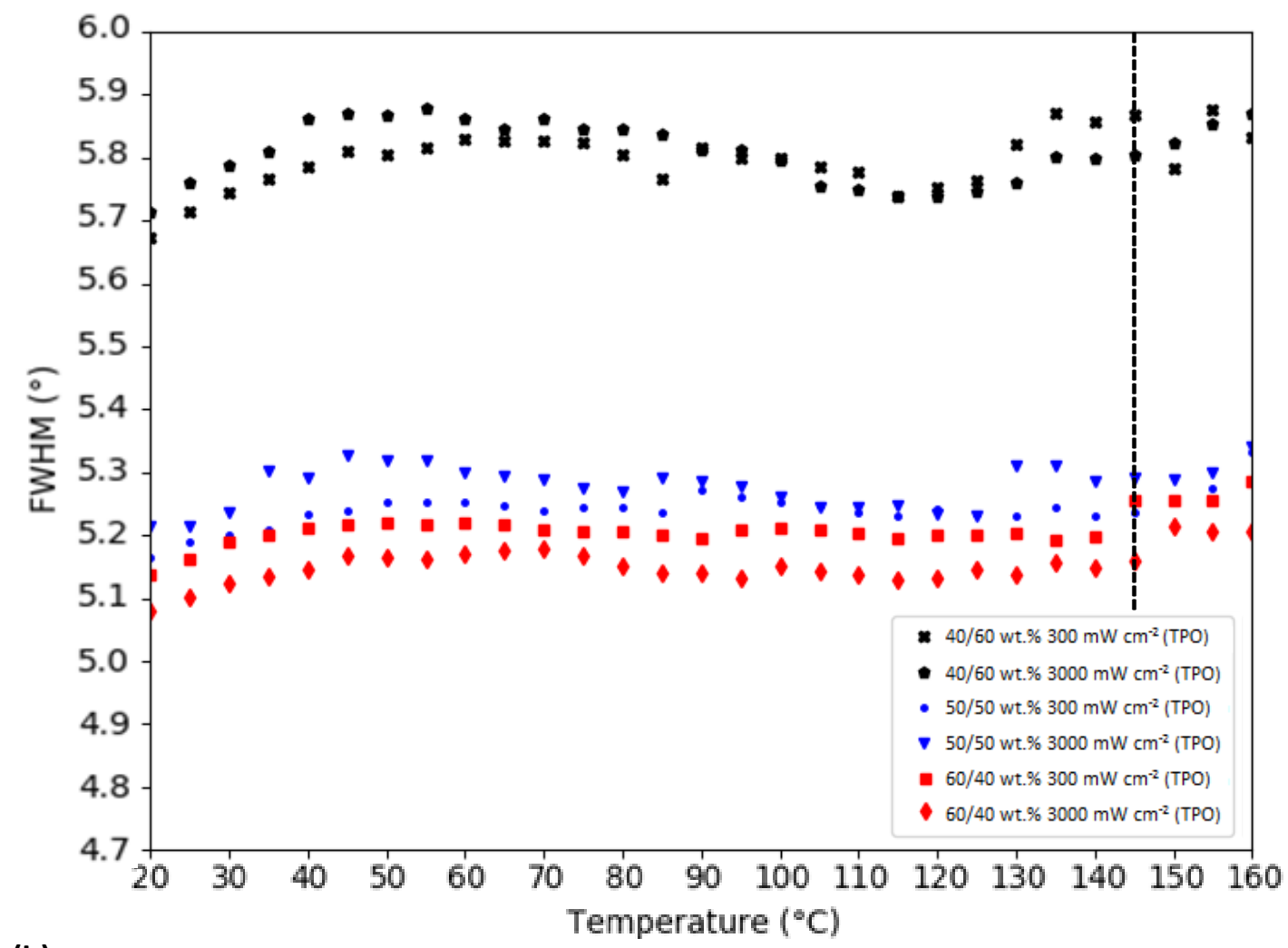

Figure S3. FWHM of the principal scattering peak as a function of temperature for (a) CQ and (b) TPO initiated resins. Data to the right of the broken vertical line represent points where the heating platform could not maintain the heating rate due to thermal losses, but where data was still collected at the respective temperatures. 